\title{
LA CONSTRUCCIÓN DEL ORGANISMO SOCIAL COMO UNA OBRA DE ARTE (REPEATING BEUYS)
}

\author{
Álex Carrascosa Vacas \\ Gernika Gogoratuz Peace Research Center
}

\section{Resumen}

Este artículo está constituido por tres partes. Una primera parte presenta la corriente del Arte-Vida (el lifelike art, en expresión de Allan Kaprow, creador del Happening) y sus dos principales tendencias: la vía Duchamp, consistente en la ampliación del campo del arte mediante la apropiación de elementos extra-artísticos, incluso la propia vida cotidiana; y la vía productivista, que por el contrario busca socializar el arte, aplicar el arte al proceso de construcción de un nuevo modelo social; para finalmente encontrar ambas tendencias, a través de Beuys y la Internacional Situacionista, en una idea común, la consideración de la sociedad como materia plástica, susceptible de ser (re)creada como una obra de arte, como una conciencia estÉtica. Una segunda parte, presenta las llamadas Tecnologías Sociales o Blandas como resultado sucesivo de la confluencia de la psicología social, la teoría psicoanalítica y la teoría de sistemas, en su intento conjunto de comprender el campo psicosocial y abordarlo en aras de la evolución de las relaciones e interacciones humanas. Y una tercera parte se centra en una de estas tecnologías sociales, el Proceso 'U', para ilustrar cómo puede contribuirse sustancialmente a la construcción del organismo social como una obra de arte.

Palabras clave: ARTE-VIDA; ESCULTURA SOCIAL; TECNOLOGÍAS SOCIALES; CAMPO RELACIONAL; ESTÉTICA; ÆFECTIVIDAD

\section{THE CONSTRUCTION OF SOCIAL ORGANISM AS A WORK OF ART (REPEATING BEUYS)}

\section{Abstract}

This article consists of three parts. The first one presents the Art-Life movement (or lifelike art, as Allan Kaprow, creator of Happening, coined) and its two main trends: the Duchampian way, which lies in the expansion of the field of art by appropriating extra-artistic elements, including everyday life; and the productivist way, which contrarily aims at socializing art by applying it to the process of constructing a new social model; and then-through Beuys and the Situationist International-both trends are joined together around a common idea, the consideration of society as plastic/sculptural matter, which can be (re)created as an artwork, as an aesthEthical consciousness. Afterwards, the second part presents the so-called Social or Soft Technologies as an ongoing result of the confluence of social psychology, psychoanalytic theory and systems theory, in their joint attempt to understand the psychosocial field and address it with the purpose of evolving human relations and interactions. And lastly, the third part focuses on one of these social technologies, the ' $U$ ' process, in order to illustrate how it can be contributed substantially to the construction of social organism as a work of art.

Keywords: ART-LIFE; SOCIAL SCULPTURE; SOCIAL TECHNOLOGIES; RELATIONAL FIELD; AESTHETHICS; ÆEFECTIVITY

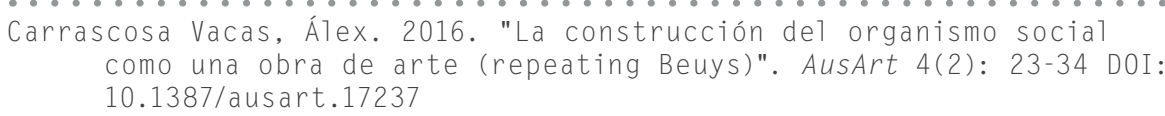

\section{AUSART}


Llevar el arte a la vida: ¿Existe utopía más persistente en la historia de las vanguardias? Lo que está en juego es algo más que una renovación estilística:

Se trata de transformar tu existencia cotidiana

(Brian Holmes).

\section{LAS CORRIENTES DEL ARTE-VIDA}

A lo largo del s. XX se suceden las propuestas por parte de muchas y muchos artistas de vanguardia de liberar al arte de sus soportes y disciplinas tradicionales y llevarlo a la vida cotidiana. Estas propuestas, que Kaprow calificaba como lifelike art —arte-como-vida o arte=vida ${ }^{1}$ —, se agrupan en diferentes tendencias dependiendo de su motivación, ya sea puramente artística, ya social o política.

Una primera tendencia, constante en la historia del arte moderno, busca apropiar para el arte elementos no artísticos, desde los objetos cotidianos hasta las actividades humanas. Partiendo de los collages cubistas (Picasso, Braque), de los ready-mades dadaístas (Duchamp) o de los objets trouvés surrealistas, un ramal despunta a través de los ensamblajes de escala habitacional (Schwitters; Nevelson) o de las acumulaciones y envolvimientos de incidencia paisajística (Christo y Jeanne-Claude); y otro descuella prolongando el lienzo a la pintura habitable (Dubuffet; Hundertwasser; Saint-Phalle), hasta encontrarse ambos en el happening o acontecimiento (Kaprow; la red Fluxus: Beuys, Lebel, Vostell...). El happening marca la transición de la integración de lo cotidiano en el arte hacia la integración del arte en lo cotidiano: en primer lugar "extiende los ambientes objetuales, donde los objetos, más que escenográficos son parte constitutiva de la acción" (Marchán [1972] 1990, 193-4); en segundo lugar interpreta trascendentemente la action painting de Pollock, cuya razón no estriba tanto en el goteo de la pintura sobre la tabla como en el movimiento del pintor en el espacio; y en tercer lugar incluye al espectador o a la espectadora para activación de su creatividad. En suma: espacio extra-artístico, artistas y audiencia configuran una entera "obra plástica". En esta corriente, el siguiente avance a partir del happening consistirá en declarar "arte" las vivencias cotidianas tanto personales - Activities de Kaprow, Maintenance Art de Ukeles - como interpersonales - happenings de Vostell o de Beuys, Encounters de Demattio, performances de Lacy-. 
Una segunda tendencia responde a motivaciones más sociales que puramente artísticas. De ésta se desprenden dos haces de líneas: unas paralelas a la apropiación de la vida para el arte, como ocurre con el happening político de Beuys; y otras independientes a dicha apropiación, como los productivismos, que más bien proceden de modo inverso, no tratando de artificar la vida, sino de socializar el arte, esto es, de aplicar el arte a la edificación de un nuevo modelo social; o como la Internacional Situacionista, según la cual las producciones artísticas se integran en una construcción cultural superior y por eso, no puede existir un arte situacionista, sino más bien un uso situacionista de los medios artísticos (Internationale Situationniste [1958-1961] 2001, 15).

La Internacional Situacionista (1958-1969), se constituye como consejo ${ }^{2}$ de trabajadoras y trabajadores de la cultura para la crítica y la renovación de la vida cotidiana a escala de laboratorio (I.S. [1958-1961] 2001, 22; 153-4). El objetivo de la I.S. es triple: en primer término propone la construcción y la vivencia de situaciones o unidades espacio-temporales de participación colectiva que restauren en toda persona la consciencia y el protagonismo sobre sí misma y la capaciten para aportar a toda acción, cualquiera que sea, un comportamiento creativo $^{3}$; en segundo término plantea, a partir de dichas situaciones, liberar vacíos - como huecos de electrón de carga positiva - a la trama jerárquica, disociada y propagandística de la ciudad (I.S. [1958-1961] 2001, 211-2) hasta conjugar la suma de artes y actitudes conscientes en una suerte de urbanismo unitario; y a la postre, trata de "'realizar' el arte, de construir el arte efectivamente, en todos los niveles de la vida" (J.V. Martin, J. Strijbosch, R. Vaneigem \& R. Viénet en I.S. [1961-1968] 2004, 160), pues la persona situacionista, en tanto que agente revolucionaria, "considera plástico su propio entorno y también a sí misma” (I.S. [1958-1961] 2001, 153).

Cerrando una espira, esta noción de plasticidad es del todo concurrente con Joseph Beuys cuando el propio artista se declara obra plástica, constitución celular y organismo que filosofa, habla y denuncia, de la misma manera que toda revolución social puede ser una "(re)evolución" a medida que la sociedad vaya progresivamente dándose forma a sí misma. Beuys abre en la Documenta 5 de Kassel (1972) una oficina informativa de la Organización para la Democracia Directa mediante Plebiscito y atiende una Conversación de 100 días (tantos como dura la exposición) con el público sobre democracia y arte. Al enmarcarse en el espacio y el tiempo del Arte institucional, su conversatorio no va más allá de ser otra obra de arte, en la tradición duchampiana de apropiación de lo extra-artístico; pero en su declaración "I am searching for field 
character", de 1973, Beuys coincide en la realización del arte proclamada por los y las situacionistas:

"El arte es ahora el único poder evolutivo-revolucionario [(re)evolucionario] capaz de desmantelar los efectos represivos de un sistema social senil para construir un ORGANISMO SOCIAL COMO OBRA DE ARTE. Esta disciplina de arte más moderna -Escultura Social / Arquitectura Social— sólo cumplirá su objetivo cuando toda persona viviente se convierta en creadora, escultora o arquitecta del organismo social. Sólo entonces el Happening se cumplirá. [...] TODO SER HUMANO ES UN ARTISTA que - desde su estado de libertad- aprende a determinar su posición en la OBRA DE ARTE TOTAL DEL ORDEN SOCIAL FUTURO". ${ }^{4}$

La "conquista de la vida cotidiana" por parte de la Internacional Situacionista (I.S. [1958-1961] 2001, 153-4) o la re-evolución artística prolamada por Beuys se enmarcan en un ciclo histórico emancipatorio jalonado por experiencias afines como los Círculos de Cultura en Brasil (1960-1964), desde los que Paulo Freire promueve la transitividad crítica o "apropiación progresiva por parte de la persona de su posición en el contexto"; o los grupos de autoconciencia de las New York Radical Women (1967), que popularizan la consigna "lo personal es político".

\section{EL SURGIMIENTO DE LAS TECNOLOGÍAS SOCIALES. 5}

Complementariamente a la (re)evolución de las artes, el aporte más reconocido al empoderamiento de las personas, a la toma de conciencia sobre sí mismas y sobre su contexto en aras de la transformación de éste, proviene de "la confluencia de la psicología social, de la teoría psicoanalítica y de la teoría de sistemas" (Bunker \& Alban 1997, 11).

El primer afluente se personifica en Kurt Lewin. En 1945, doce años después de su exilio a Estados Unidos, el psicólogo gestáltico de origen alemán co-funda y dirige el Centro de Dinámicas de Grupo en el Instituto Tecnológico de Massachusetts (Boston). Allá lleva a cabo un estudio científico de los procesos que influyen en las personas cuando son parte de un grupo y crea una primera tecnología social, el sensitivity training group (grupo de terapia), más conocido como "T group". A través de los "T groups" Lewin desarrolla tanto 
la investigación-acción (forma de indagación científica realizada desde una comunidad de práctica), como la Teoría del Campo (Field Theory, 1951), según la cual los individuos (y grupos) son influidos por el campo dinámico de fuerzas o espacio vita/ ${ }^{6}$ en el que se encuentran.

En 1947, con la muerte de Lewin, el Centro de Centro de Dinámicas de Grupo se traslada a la Universidad de Michigan, donde pasa a llamarse Instituto de Investigación Social. Ese mismo año, el alumno de Lewin Ron Lippitt funda junto con Lee Bradford y Ken Benne el Instituto NTL sobre Ciencias Aplicadas del Comportamiento. Durante los años 1950 y 1960, el campus de verano del NTL en Bethel (Maine) es un semillero de aprendizaje experimental (Bunker \& Alban 1997, 15). Además de las dinámicas de grupo, se estudia el funcionamiento de las tareas - cómo se toman las decisiones, cómo se resuelven los problemas y se planean e implementan las acciones-. Lippitt diseña actividades que ayudan a los grupos a pensar en el camino hacia su "futuro preferido" imaginando sus organizaciones cinco años más tarde, sobrevolándolas y observando desde arriba qué cosas maravillosas están sucediendo; y de aquí descubre cómo pensar en lo que se quiere infunde energía en las personas (Bunker \& Alban 1997, 16-7).

Volviendo al MIT, Edgar Henry Schein (referente en el desarrollo organizacional) crea entre los años 60 y 70 un nuevo modelo de aprendizaje que sitúa a la persona alumna como conductora del cambio y a la educadora como coach (mentora o entrenadora). Mientras tanto, en el mismo edificio universitario, Jay Forrester desarrolla las primeras computadoras digitales mediante la interacción de diferentes secuencias paramétricas: crecimiento poblacional, producción alimentaria, desarrollo industrial, contaminación y consumo de recursos naturales no renovables. A partir de estas simulaciones de la Tierra como un sistema integrado, Donella Meadows co-crea el 'modelo World3', que constituye la base del informe encargado por el Club de Roma Limits to Growth (1972). Este libro predice hace casi 45 años un colapso que ha corroborado recientemente la Universidad de Melbourne?: "si las actuales tendencias de crecimiento de la población mundial, la industrialización, la contaminación, la producción de alimentos, y el agotamiento de los recursos siguen sin cambios, los límites al crecimiento en este planeta se alcanzarán en algún momento dentro de los próximos cien años. El resultado más probable será un declive bastante repentino e incontrolable en la población y la capacidad industrial" (Meadows et al. 1972, 23). 
Al equipo de Jay Forrester se une el científico de sistemas Peter Senge, que dirige el Centro de Aprendizaje Organizacional del MIT. El interés de Senge reside en vincular las dinámicas del sistema con un cambio mundial real: "la esencia del pensamiento aplicado a los sistemas es ayudar a la gente a cerrar el bucle de retorno entre la representación conductual [visible pero inconsciente] de los sistemas de los que forma parte, y su fuente invisible de consciencia y de pensamiento" (Scharmer 2009, 53, 135); dicho de otro modo, la observación consciente de cómo recreamos el propio sistema que nos afecta.

Y complementariamente a Senge, en los años 80, Marvin Weisbord hace hincapié en la importancia de "tener todo el sistema en la habitación" ("getting the whole system into the room"). Weisbord crea un nuevo método, Future Search (la Búsqueda de Futuro), al que suceden, durante las décadas posteriores, las principales tecnologías sociales (sistematizadas como Large Group Interventions o intervenciones en grupos grandes por Barbara Bunker y Billie Alban). Éstas tienen en común el abordaje de desafíos y problemas de forma integral, involucrando a una masa crítica de personas y permitiéndolas participar en "el análisis de su realidad actual, en la generación de ideas sobre la transformación de los procesos existentes, y en la implementación y realización de dicha transformación" (Bunker \& Alban 1997, xvi).

Muchas de las nuevas tecnologías sociales o blandas responden tanto al sector comercial como al no lucrativo; tal es el caso de la Indagación Apreciativa (Cooperrider, Srivastva y Whitney 1987-2001), del Open Space (Owen 1992), del World Café (Brown e Isaacs 1995) o de la Teoría U o Laboratorio del Cambio (Scharmer y Jaworski 2000). En el caso de la Planificación de Escenarios (Kahn, años 60), si bien este método se relaciona inicialmente con la inteligencia militar, es significativamente utilizado durante el proceso de Mont Fleur en Sudáfrica (Kahane, 1990-1994), un diálogo multiactor orientado a la recreación del país durante los futuros 10 años. Otras tecnologías son exclusivamente creadas para la innovación social. Algunas, como la Democracia Profunda (Mindell, 1988), el Diálogo de Bohm (publicado en 1996), el Diálogo Sostenido (Saunders, creado en los 60 e implementado en los 90) y el Círculo de PeerSpirit (mediados de los 90 ), se orientan al trabajo relacional; y otras, como el Proceso de Planificación Estratégica (ICA, Institute of Cultural Affairs International, finales de los 80) y el Diseño Centrado en las Personas (IDEO, 2009), al desarrollo comunitario. 


\section{EL PROCESO ' $U$ ' $Y$ LA PRESENCIACIÓN}

Recogiendo los sucesivos testigos de Lewin, Schein, Forrester y Senge, y con la ayuda de Jaworski, Käufer, Nonaka y otros, Otto Scharmer ${ }^{8}$ crea el proceso 'U' (más conocido como Teoría $U$ ), una tecnología social consistente en cinco fases o movimientos diferentes (Scharmer 2009, 18-9, 314):

1. Co-Iniciación: Conocimiento mutuo de las y los diferentes actores en un determinado sistema.

2. Co-Percepción: Viajes de aprendizaje y sentido a los lugares de mayor potencial; y al mismo tiempo, cultivo recíproco de la escucha empática (desde el lugar de la persona que habla).

3. Co-Presenciación: Retiro y reflexión para permitir que el conocimiento interior emerja; para soltar apegos y resistencias individuales, y reconocer y acoger la más alta posibilidad como colectivo.

4. Co-Creación: Cristalización de ideas y prototipado de los primeros modelos de intervención en la realidad con probatura y feedback inmediatos.

5. Co-Evolución: Desarrollo y mejora progresiva de los modelos de innovación.

De estas cinco fases, la Presenciación (Presencing en inglés) constituye el punto de inflexión del proceso U. Dicho concepto procede de la fusión de 'presence' (entendido como mindfulness o conciencia plena sobre las experiencias internas y externas que concurren en el momento presente) y 'sensing' (percepción sensible, sintonización con nuestro máximo potencial futuro), y remite a la conexión con la fuente de nuestra intención y atención (Scharmer 2009, 06-08), allá donde se generan nuestras acciones.

Para entender mejor este término, Scharmer invita a observar del trabajo del/la artista desde tres ángulos distintos: 1) el objeto resultante del proceso creativo (el cuadro); 2) el proceso de pintar el cuadro; y 3) el o la artista ante el lienzo en blanco. Expresado en dimensiones temporales: el después (objeto); el durante (proceso); y el antes (lienzo en blanco y origen de la acción creadora). La Presenciación funciona precisamente como un instrumento de auto-observación de la fuente donde se origina nuestra intención y nuestra atención, un telescopio que se vuelve sobre la o el astrónomo. 
Y así como el telescopio está ideado para examinar la bóveda celeste, la Presenciación está ideada para contemplar el campo social. Scharmer, siguiendo la tradición lewiniana, atribuye al campo dos aspectos: el visible (sobre la superficie) y el invisible (bajo ésta). En los campos sociales la materia visible es lo que hacemos, decimos y vemos; y el mundo invisible, el estado interior desde el que los participantes de una situación actúan. La esfera intermedia que une ambas dimensiones es la estructura del campo de la atención.

El instrumento de la Presenciación se acompaña de una doble guía para ayudar a cada persona en la observación de sí misma y su entorno relacional e interaccional. Dicha guía enuncia cuatro lugares posibles en el campo de la atención y cuatro tipos básicos de escucha asociados a cada lugar. Asimismo, el campo de atención no se limita a un solo órgano perceptivo, la mente; sino que se amplía al corazón y a la voluntad (Scharmer 2009, 11-3): head, heart and hand, en relación con las tres haches del aprendizaje experiencial de John Dewey ${ }^{9}$.

- Nivel 1, Yo-en-mí: La escucha se instala en la descarga. Todo cuanto la persona escucha es filtrado por su sistema de creencias y supuestos y confirma lo que ésta ya sabía.

- Nivel 2, Yo-en-ello: La escucha se basa en la diferencia. Se desconfirman algunos supuestos. Ahora bien, la actitud puede ser reactiva (defensa, debate o rebatimiento); o indagadora (cuestionamiento).

- Nivel 3, Yo-en-ti: La escucha es empática, desde el lugar de la otra persona.

- Nivel 4, Yo-en-ahora: La escucha es generativa; se sitúa en el campo emergente del futuro. Las personas en diálogo comparten un estado alterado de "comunión" o "gracia".

\section{CONCLUSIÓN}

Según Scharmer, la crisis de nuestro tiempo revela el fin de una mentalidad y una estructura institucional y social obsoletas, y al mismo tiempo, la emergencia de una nueva forma de presencia y poder que crece espontáneamente a través de redes y colectivos pequeños. Al operar desde una posibilidad futura real, los grupos acceden a un campo social diferente, donde las personas pue- 
den superar los patrones del pasado y conectar con una fuente de creatividad y de conocimiento más profunda (2009, 01-04). El objetivo de la Presenciación y del entero Proceso 'U', como ejemplo paradigmático de tecnología social, es precisamente la atención simultánea a la dimensión de la acción visible y de la intención invisible (08-10). La toma de conciencia, hacia adentro, sobre los lugares interiores desde donde actuamos en tiempo real, y la auto-observación, hacia afuera, desde cada quien y en grupo, del sistema que inconscientemente recreamos y cuyos resultados nos afectan, pueden marcar el punto de inflexión para una transformación æfectiva y creactiva de nuestro entorno.

Si bien la evolución del campo artístico y el desarrollo del campo psicosocial discurren independientemente, es plausible la vinculación de ambos: la aplicación del arte al desenvolvimiento de los procesos y sinergias grupales y sociales y viceversa, la utilización de las tecnologías sociales o blandas (referidas a las relaciones e interacciones humanas) en el modelado de la sociedad como materia plástica, susceptible de ser (re)creada como una obra de arte, como una conciencia estÉtica.

Desde el formato del taller, artes visuales y tecnologías blandas pueden fundirse en un proceso orgánico. Genéricamente, el colectivo - grupo de trabajo, comunidad de práctica, consejo o asamblea- encarnaría un proceso de transformación consistente en tres momentos básicos: (re)conocimiento sensible del medio; auto-observación; y diseño de acciones. En primer lugar, las personas se capacitan en la escucha activa mutua y común, así como en la decantación y plasmación de la información cualitativa, no tanto proveniente del análisis mental e ideológico, sino de la experiencia sensible. En segundo lugar, una vez (re)conocidos los intereses del conjunto de las personas, se concientiza la presencia en el aquí y en el ahora: cada cual identifica aquellas resistencias que le impiden avanzar y acoge después la más alta posibilidad para el conjunto. $Y$ en tercer lugar, se cristalizan las ideas emergentes y se modelan escenarios y acciones de futuro. Fuera del taller, las acciones diseñadas se implementan en la realidad, recibiendo respuesta inmediata del contexto y siendo mejoradas cada vez. De este modo, el grupo de trabajo ayuda al organismo social a darse forma como una creación plástica o incluso como una obra de arte, si atendemos a los prototipos de futuro y a su activación. Llevando el sistema a la habitación, y la habitación al sistema, transformando hábitos y patrones, el grupo puede contribuir directamente a la co-creación de la obra de arte total del orden social futuro. 


\section{Referencias}

A.F.R.I.K.A. Gruppe et al. 2001. Modos de hacer: Arte crítico, esfera pública y acción directa. Paloma Blanco, Jesús Carrillo, Jordi Claramonte \& Marcelo Expósito, eds. Salamanca: Universidad de Salamanca

Bojer, Marianne, Marianne Knuth \& Colleen Magner. 2006. Mapping dialogue: A research project profiling dialogue tools and processes for social chang. Johannesburgo: Pioneers of Social Change

Bookchin, Natalie et al. 2013. Militant Research Handbook. New York: New York University

Bunker, Barbara Benedict \& Billie T. Alban. 1997. Large group interventions: Engaging the whole system for rapid change. San Francisco CA: Jossey-Bass

Carrascosa Vacas, Álex. 2010. Dia-Tekhnē: Diálogo a través del arte. Bilbao: Bakeaz; Gernika Gogoratuz

- 2014. "On The 'Dia-Tekhnē - Dialogue Through Art' Methodology". In Factis Pax 8(1): 62-91. http://www.infactispax.org/volume8dot1/Carrascosa.pdf

Castoriadis, Cornelius. 2005. Escritos políticos. Edición de Xavier Pedrol. Madrid: Los Libros de la Catarata

Debord, Guy. (1967) 1995. La sociedad del espectáculo. Traducción: Rodrigo Vicuña Navarro. Santiago de Chile: Ediciones del Naufragio

Dewey, John. (1934) 2008. El arte como experiencia. Traducción y prólogo de Jordi Claramonte. Barcelona: Paidós

Egbert, Donald Drew. 1981. El arte y la izquierda en Europa: De la Revolución francesa a Mayo de 1968. versión del inglés de Homero Alsina Thevenet. Barcelona: Gustavo Gili

Freire, Paulo. 1965. Educación como práctica de la libertad. Bogotá: América Latina

Fromm, Erich. (1978) 1995. ¿Tener o ser?. Traducción de Carlos Valdés. México DF: Fondo de Cultura Económica

González García, Ángel, Francisco Calvo Serraller \& Simón Marchán Fiz, eds.. 1979. Escritos de arte de vanguardia 1900/1945. Madrid: Turner

Hannah Arendt. 1988. Sobre la revolución. Versión española de Pedro Bravo. Madrid: Alianza

Holmes, Brian. 2011. “'Eventwork': La cuádruple matriz de los movimientos sociales contemporáneos". https://brianholmes.wordpress.com/2012/02/17/eventwork/

Home, Stewart. 2002. El asalto a la cultura: Corrientes utópicas desde el Letrismo a Class War. Traducción del inglés, Jesús Carillo y Jordi Claramonte. Barcelona: Virus

IDEO. 2011. Human-centered design toolkit: An open-source toolkit to inspire new solutions in the developing world. Palo Alto CA: IDEO

Internationale Situationniste. (1958-1961) 2001. Internacional Situacionista: Textos íntegros en castellano de la revista Internationale Situationniste (1958-1969). Vol. 1, La realización del arte: Textos de IS 1-6 (1958-1961). Traducción, Luis Navarro. Madrid: Literatura Gris

- (1961-1968) 2004. Internacional Situacionista:Textos íntegros en castellano de la revista Internationale Situationniste (1958-1969). Vol. 2, La supresión de la política: Textos de IS 7-10. Traducción, Luis Navarro. Madrid: Literatura Gris

- 1969. Numéro 12 (septembre). http://www.larevuedesressources.org/IMG/pdf/internationale_situationniste_12.pdf 
Kaprow, Allan. (1993) 2003. Essays on the blurring of Art and Life. Berkeley CA: University of California

- 2007. La educación del des-artista. Edición y traducción, Armando Montesinos y David García Casado. Madrid: Árdora

Marchán Fiz, Simón. (1972) 1990. Del arte objetual al arte de concepto. Madrid: Akal

Meadows, Donella, H. Dennis L. Meadows, Jørgen Randers \& William W. Behrens III. 1972. The limits to Growth: A report for the Club of Rome's project on the predicament of Mankind. New York: Universe Books

Mindell, Arnold. 2004. Sentados en el fuego. Cómo transformar grandes grupos usando el conflicto y la diversidad. Trad., José Luis Escorihuela. Barcelona: Icaria

Murray, Robin, Julie Caulier-Grice \& Geoff Mulgan. 2010. The Open Book of Social Innovation. London: The Young Foundation. http://www.nesta.org.uk/sites/default/files/the_open_ book_of_social_innovation.pdf

Nance, Claire. 1991. The individual as a channel of group process: Case studies in group process work. Seattle: Antioch University. http://www.processwork.org/files/Finalprojects/ Nance-Groups.pdf

Parreño Velasco, José María. 2006. Un arte descontento: arte, compromiso y crítica cultural en el cambio de siglo. Murcia: Cendeac

Popper, Frank. 1989. Arte, acción y participación: El artista y la creatividad hoy. Traducción de Eduardo Bajo. Madrid: Akal

Rolnik, Suely. 2007. "La memoria del cuerpo contamina el museo". Número monográfico "Arte y Revolución". Brumaria 8

Scharmer, Claus Otto \& Katrin Käufer. 2013. Leading from the emerging Future: From ego-system to eco-system economies. EBSCO Academic eBook. San Francisco CA: Berrett-Koehler

Scharmer, Claus Otto. 2009. Teoría U: Liderar desde el futuro a medida que emerge. Traducido del inglés por Montserrat Foz Casals. Barcelona: Eleftheria

Stachelhaus, Heiner. 1990. Joseph Beuys. Traducción Joan Godo Costa. Barcelona: Parsifal

Tisdall, Caroline, ed. 1974. Art into Society, Society into Art: seven german artists (29 October 24 November 1974). London: ICA

Viénet, René. (1968) 1978. Enragés y situacionistas en el movimiento de las ocupaciones. Traductor J. Gómez. Madrid: Castellote. http://www.sindominio.net/ash/enrages.htm

\section{Notas}

${ }^{1}$ Arte-como-vida según la traducción de Armando Montesinos y David García Casado, y arte=vida según la expresión de Simón Marchán-Fiz.

${ }^{2}$ La Internacional Situacionista se inspira en el Consejismo. Ejemplos de consejos son la Comuna de París de 1871; los sóviets o asambleas autónomas de obreros, campesinos y soldados surgidos en la Revolución rusa de 1905 y oficializados en la Revolución de febrero de 1917; los räte o consejos de la Revolución de Noviembre de 1918 en Alemania; o los munkástanácsok o comités obreros de la Revolución húngara de 1956. 
${ }^{3}$ Textos íntegros en castellano de la revista Internationale Situationniste (1958-1969), vol. I: La realización del arte: I.S. \# 1-6, Madrid, Literatura Gris, 2001, pp. 12, 21, 196-198; y vol. ॥: La supresión de la política: I.S. \# 7-10, Madrid, Literatura Gris, 2004, pp. 108, 146.

${ }^{4}$ «I am searching for field character», declaración de Beuys de 1973, publicada por primera vez en Caroline Tisdall, Art into Society, Society into Art (ICA, Londres, 1974), p .48. Puede verse el texto íntegro en: http://greenlanternpress.wordpress.com/2009/06/07/i-am-searching-for-field-character/

${ }^{5}$ Se llama Tecnología Social a la aplicación del conocimiento científico no a la materia inerte, sino a los sistemas sociales; este concepto es sinónimo de Tecnología Blanda, es decir, el conjunto de técnicas y métodos aplicados al desarrollo de las relaciones e interacciones humanas.

${ }^{6}$ Para Albert Einstein "la totalidad de los hechos coexistentes que son concebidos como mutuamente interdependientes se denomina 'campo'” (1905). Kurt Lewin construye su Teoría del Campo a partir de esta definición. Según Lewin (1951), la comprensión del comportamiento de un individuo o de un grupo implica comprender la totalidad de las fuerzas que operan en el campo psicológico, el campo de fuerza o espacio vital; o dicho de otro modo: el «entorno personal y psicológico tal como existe para cualquier individuo incluyendo sus necesidades, motivaciones, propósitos, preocupaciones e ideales». «De acuerdo con la teoría del campo, el comportamiento no depende del pasado ni del futuro, sino del presente, que tiene una determinada profundidad temporal. En el presente, los componentes del pasado y del futuro psicológico son simultáneos» (Nance 1991). Más recientemente (1995), Arnold Mindell, desde otra tecnología social, el Process Work (o psicología orientada a procesos, para el tratamiento personal, interpersonal y grupal de las dimensiones visibles y ocultas en un conflicto), desarrolla el concepto campo, definiéndolo como «la atmósfera de un grupo, su humedad, sequedad, tensiones y tormentas; atmósfera que recorre nuestro ser individual, abarca grupos enteros, organizaciones, ciudades y el medio ambiente». Añade: «el campo se puede sentir; es hostil o amoroso, reprimido o fluido; no sólo se compone de estructuras abiertas, visibles y tangibles, como agendas, plataformas y debates, sino también de procesos emocionales, ocultos, invisibles e intangibles, como los celos, los prejuicios, el dolor o la ira» (Mindell 2004, 25).

${ }^{7}$ https://www.theguardian.com/commentisfree/2014/sep/02/limits-to-growth-was-right-new-research-shows-were-nearing-collapse

${ }^{8}$ Dr. C. Otto Scharmer, economista y profesor de la Sloan School of Management del MIT; creador junto a Joseph Jaworski del Change Lab, posteriormente conocido como la Teoría 'U'; co-fundador del Presencing Institute.

9 John Dewey (1859-1952) consideraba el arte como eje del sistema educativo; se trataba de combinar el estudio, la vida comunitaria y el trabajo manual. Head, heart and hand fueron asimismo los principios del Black Mountain College (BMC), pequeña universidad experimental y punto de encuentro de John Cage, que desarrolla su concepto ampliado de música y sus primeras performances, del bailarín y coreógrafo contemporáneo Merce Cunningham, de los pintores Willem y Elaine de Kooning, Robert Rauschenberg y Cy Twombly, de Buckminster Fuller, Kenneth Snelson y su primer Domo geodésico, y de Allan Kaprow y su primer prototipo de happening conocido como event (evento) (Carrascosa 2010, 42-5). 